\title{
Malignant mixed Müllerian tumor of the ovary
}

INSERM

\section{Source}

INSERM. (1999). Orphanet: an online rare disease and orphan drug data base. Malignant mixed Müllerian tumor of the ovary. ORPHA:213512

Malignant mixed Müllerian tumor of the ovary is a rare and very aggressive neoplasm presenting most commonly in postmenopausal women and is composed of adenocarcinomatous and sarcomatous elements and, depending on the types of these elements, can be classified as homologous or heterologous. It often has a poor prognosis. 